\title{
Single-locus complementary sex determination in the inbreeding wasp Euodynerus foraminatus Saussure (Hymenoptera: Vespidae)
}

\author{
JK Stahlhut and DP Cowan \\ Department of Biological Sciences, Western Michigan University, Kalamazoo, MI 49008-5410, USA
}

The Hymenoptera have arrhenotokous haplodiploidy in which males normally develop from unfertilized eggs and are haploid, while females develop from fertilized eggs and are diploid. Multiple sex determination systems are known to underlie haplodiploidy, and the best understood is singlelocus complementary sex determination (sl-CSD) in which sex is determined at a single polymorphic locus. Individuals heterozygous at the sex locus develop as females; individuals that are hemizygous (haploid) or homozygous (diploid) at the sex locus develop as males. SI-CSD can be detected with inbreeding experiments that produce diploid males in predictable proportions as well as sex ratio shifts due to diploid male production. This sex determination system is considered incompatible with inbreeding because the ensuing increase in homozygosity increases the production of diploid males that are inviable or infertile, imposing a high cost on matings between close relatives. However, in the solitary hunting wasp Euodynerus foraminatus, a species suspected of having sl-CSD, inbreeding may be common due to a high incidence of sibling matings at natal nests. In laboratory crosses with $E$. foraminatus, we find that sex ratios and diploid male production (detected as microsatellite heterozygosity) are consistent with sI-CSD, but not with other sex determination systems. This is the first documented example of Sl-CSD in a hymenopteran with an apparent natural history of inbreeding, and thus presents a paradox for our understanding of hymenopteran genetics.

Heredity (2004) 92, 189-196, advance online publication, 3 December 2003; doi:10.1038/sj.hdy.6800394

Keywords: sex determination; haplodiploidy; Hymenoptera; microsatellites

\section{Introduction}

The insect order Hymenoptera, which includes wasps, ants, and bees, has a haplodiploid genetic system in which females normally develop from fertilized eggs and are diploid, while males develop from unfertilized eggs and are haploid, carrying only a single set of maternal chromosomes. However, various underlying sex determination systems, rather than ploidy level alone, determine sex in these insects. The best known mechanism is single-locus complementary sex determination, or sl-CSD (Whiting, 1943), in which heterozygotes at a single sex locus develop as females, while hemizygotes (normal haploids) and homozygous diploids develop as males. Other proposed mechanisms include multilocus complementary sex determination, or ml-CSD (Crozier, 1971), in which several loci control sex determination and only a homozygote at all sex loci would develop as a diploid male; genomic imprinting (Beukeboom, 1995; Dobson and Tanouye, 1998), in which a paternal locus carries an active female-development factor that is inactive or absent in the maternal genome; and genic balance (Bridges, 1925; Kerr and Nielsen, 1967) in which a dose-independent 'maleness' gene causes male development in haploids while a separate, dose-dependent

Correspondence: DP Cowan, Department of Biological Sciences, Western Michigan University, Kalamazoo, MI 49008-5410, USA.

E-mail: cowan@wmich.edu

Received 26 February 2003; accepted 18 July 2003 'femaleness' gene causes individuals of higher ploidy to develop as females.

These sex determination hypotheses can be distinguished based on the predictions they generate regarding the offspring of consanguineous matings. Complementary sex determination, whether controlled by a single locus or more than one, can be distinguished from other sex determination systems by an increase in males among the offspring of sibling matings. This is due to the presence of diploid males, which are produced in predictable proportions and can be shown to be heterozygous (and therefore confirmed diploid) via genetic markers. Under noncomplementary systems such as genic balance or genomic imprinting, diploid males will normally be absent, and inbreeding will not result in sex ratio shifts (Cook, 1993b).

Although the genomic imprinting hypothesis has received empirical support in some species (Dobson and Tanouye, 1998), the genic balance model has not been supported for Hymenoptera by experimental evidence (Cook, 1993b; MacDougall et al, 1995). It is important to note that the mere presence of diploid males does not confirm that sex determination is complementary. A rare mutant polyploid strain of Nasonia vitripennis is known to produce diploid males, but, like normal haploids, these diploid males develop only from unfertilized eggs; in this case, the eggs producing diploid males are themselves diploid (Whiting, 1960; Dobson and Tanouye, 1998). There are two important distinctions between diploid male production under CSD and diploid male production under a 
noncomplementary system such as genomic imprinting. First, noncomplementary models predict no association between inbreeding and diploid male production, so that males should account for the same proportion of diploids among inbred and outbred sibships. Second, any diploid males that may occur under noncomplementary systems are predicted to carry only maternal genetic material, since they can develop only from unfertilized diploid eggs, or from fertilized diploid eggs which have lost their paternally derived chromosomes. In order to support the presence of CSD, diploid males must (a) occur in inbred broods in proportions predicted by an sl-CSD or mlCSD model, (b) be absent from outbred broods (except in the rare case of a mating between nonrelatives with a shared sex allele, described below), and (c) inherit segregating genetic material from both parents.

Random mating in a population with sl-CSD occasionally results in a union between a male and female that share a sex allele. This is called a matched mating (Adams et al, 1977). The sex locus is expected to be highly polymorphic within populations, and, under random mating, the equilibrium frequency of each of $k$ sex alleles will be equal to $1 / k$ (Yokoyama and Nei, 1979). Thus, the overall chance of any mating in the population being matched is 2/k (Cook and Crozier, 1995; Adams et al, 1977). In matched matings, $50 \%$ of the diploid offspring are predicted to develop as males instead of females, so under random mating the proportion of diploids that are male will be equal to $50 \%$ of $2 / k$, or $1 / k$. Previous estimates of $k$ in natural hymenopteran populations have ranged from 11 to 80 , so diploid males may be expected to constitute between $1 \%$ and $9 \%$ of diploids under random mating (Cook and Crozier, 1995).

Diploid male production under sibling mating is expected to be much higher. Brothers and sisters have a $50 \%$ chance of sharing a sex allele, regardless of the number of sex alleles in the population; therefore, half of all sibling matings will be matched. Because half of the diploid offspring of a matched mating will develop as males, there is a $25 \%$ chance that a diploid offspring of any sibling mating will develop as a male.

Under ml-CSD, expected proportions of diploid males will be smaller than those expected under sl-CSD, and will vary as a function of the number of loci involved. The simplest case of ml-CSD is the two-locus type, in which a diploid must be homozygous at two separate sex loci to develop as a male, and therefore only a mating between individuals matched at both loci can produce diploid sons. In this case, one-fourth of sibling matings will be matched at both loci. Within these matings, only one-fourth of diploid offspring will be homozygous at both loci and develop as males, so that among all offspring of sibling matings, only one-sixteenth $(6.25 \%)$ of diploids should be male under two-locus CSD. These predicted proportions become even smaller under hypotheses of more than two sex loci. If an observed proportion of diploid males produced under inbreeding is consistent with sl-CSD, but too high to support the presence of two-locus CSD, then all forms of CSD involving more than two loci can be ruled out as well.

sl-CSD has been documented in more than 40 species distributed widely over the major taxonomic subgroups of the order Hymenoptera (Smith and Wallace, 1971; Cook, 1993b; Beukeboom, 1995), and is the prevalent system in the clade that includes the Ichneumonoidea
(Whiting, 1943; Butcher et al, 2000b) and the aculeate (stinging) Hymenoptera (Mackensen, 1950; Ross and Fletcher, 1985; Duchateau et al, 1994). The wide distribution of sl-CSD across multiple clades suggests that it may be an ancestral character state in Hymenoptera (Cook, 1993b). Although sl-CSD is often present in closely related species, such as six species in the ichneumonid genus Diadegma (Butcher et al, 2000a), it cannot be assumed for an entire higher taxon merely by its presence in one or more species in that taxon. Exceptions are known both within the aculeate-ichneumonoid clade and in other hymenopteran taxa. Cook (1993a) demonstrated that the aculeate Goniozus nephantidis (Bethylidae) lacks sl-CSD, and Beukeboom et al (2000) showed that sl-CSD is not the sex determination system of either of the braconids Asobara tabida and Alysia manducator (superfamily Ichneumonoidea), despite its presence in other braconids (Whiting, 1943; Cook, 1993b). Thus, sex determination among hymenopterans appears to be evolutionarily labile.

Diploid males in species with CSD are generally considered a genetic dead end. They may have low viability (Whiting, 1943; Petters and Mettus, 1980), may survive but be unable to mate (Smith and Wallace, 1971), or may be sterile (Cook, 1993b; Krieger et al, 1999). Spermatogenesis in male hymenopterans generally does not include a reductional division (Hogge and King, 1975); consequently, diploid males in some species are known to produce abnormal diploid sperm (Woyke and Skowronek, 1974; Yamauchi et al, 2001), which, if capable of fertilization, results in sterile triploid offspring (Inaba, 1939; Smith and Wallace, 1971; Krieger et al, 1999). The fitness of diploid males is therefore expected to be zero.

Because inbreeding increases the frequency of diploid males under any type of CSD, and because sl-CSD imparts the highest risk of diploid male production under inbreeding, we would expect selection for inbreeding avoidance in sl-CSD species. Conversely, there should be selection against sl-CSD in species whose life histories include high levels of inbreeding. Heretofore, the available data have supported these predictions (Cook and Crozier, 1995).

The behavior of the solitary hunting wasp Euodynerus foraminatus presents a paradox: facultative inbreeding in a species suspected of having sl-CSD. Field observations of this species suggest that brother-sister matings are common. Females nest in cavities such as hollow twigs, in which they provision offspring sequentially in a linear series of cells. Within a nest cavity, mothers usually first provision an uninterrupted series of daughters in the innermost cells, followed by a series of sons in the outer cells. Males develop more quickly than females, emerge from the nest first, and wait at the nest entrance to copulate with emerging sisters. Approximately $40 \%$ of females have been observed to mate with a brother before dispersal from the natal nest site (Cowan, 1979). Females become sexually unreceptive immediately after mating and will reject further mating attempts by any male (Cowan, 1986). Females not inseminated as they emerge from the natal nest mate at flowers with males that are unlikely to be related. Apparently, significant inbreeding occurs even though opportunities for outbreeding are available; we would therefore predict that E. foraminatus lacks sl-CSD. 
Inside a nest, the sequence of females is normally uninterrupted, but males are occasionally observed positioned between two sisters. These 'out-of-order' males receive the allotment of food that is normal for a developing daughter but about $70 \%$ greater than that allocated to a haploid son (Cowan, 1981). Out-of-order males may be diploids that developed from fertilized eggs, an indication of CSD. Alternatively, they may simply be haploids that developed in anomalous nest positions because either fertilization failed or the mother withheld sperm.

In order to test for the presence of CSD and differentiate between a single-locus and a multiple-locus system, we must be able to (a) detect sex ratio differences between inbred and outbred broods that are consistent with expectations under either sl-CSD or ml-CSD, (b) identify diploid (heterozygous) males using codominant genetic markers, and (c) demonstrate the presence of both maternal and paternal alleles in diploid males. To test these hypotheses in E. foraminatus, we conducted laboratory breeding experiments, compared sex ratios between experimental (sib-mated) and control (outbred) sibships, genotyped parents and offspring using microsatellites, and then compared broods produced from matched and unmatched sibling matings. For sibling and nonsibling mates, we also compared copulation durations, tendency for females to nest, and numbers of nests, cells provisioned, and surviving offspring per female.

\section{Materials and methods}

\section{Collection, breeding, and rearing of wasps}

We obtained breeding stock (parental generation wasps) from nature by using artificial nesting cavities made from sticks of wood with drilled holes (Krombein, 1967), and placing them where wild females could locate and use them as nest sites. Overwintering broods of $E$. foraminatus were collected from six sites in southwest Michigan (Allegan, Barry, and Kalamazoo Counties) in the fall of 1995. In the spring of 1996, pupae were sexed and placed in separate vials to complete development to adulthood. At maturation, virgin females were caged for mating with either a nestmate (brother) or a male collected from a different site. All matings took place between 28 May 1996 and 1 June 1996. In this parental $\left(\mathrm{F}_{0}\right)$ generation, 16 sibling (experimental group) matings and 19 outbred (control group) matings were observed. Copulation durations were timed, beginning when the male mounted the female and ending when the pair unlinked their genitalia. Males were frozen at $-80^{\circ} \mathrm{C}$ after mating. Females were maintained in $30 \mathrm{~cm}$ by $30 \mathrm{~cm}$ by $30 \mathrm{~cm}$ screened rearing cages in a greenhouse with ambient temperature and photoperiod. Each female was provided with three nesting sticks with dimensions of $1.9 \mathrm{~cm}$ by $1.9 \mathrm{~cm}$ by $9.0 \mathrm{~cm}$. Each stick had a $0.7 \mathrm{~cm}$ diameter hole drilled to a depth of $7.5 \mathrm{~cm}$. Females were also supplied with water, honey, clay soil for making cell partitions, and prey items for provisioning cells (Chilcutt and Cowan, 1993). Prey items were eastern spruce budworm larvae (Choristoneura fumiferana) that had been reared on artificial diet (Bio-Serv Inc., Frenchtown, NJ, USA). As females nested, we removed the completed nests and replaced them with empty nest sticks. Nesting females were maintained until mid-August, when the experiment was terminated and all nesting females were frozen at $-80^{\circ} \mathrm{C}$.

Completed nests were first opened several weeks after they were closed by the mother, and inspected regularly afterward to monitor brood development. Developing wasps were sexed at the pupal stage, and, at maturity, each of these $F_{1}$ offspring was weighed and then frozen at $-80^{\circ} \mathrm{C}$

\section{Microsatellite genotyping}

DNA isolations and microsatellite development were carried out as described by Stahlhut et al (2002). Fragment analysis was performed on a Beckman Coulter CEQ 2000. All parents were genotyped at seven microsatellite loci, of which five were variable and four were informative for this study. Within each family, offspring were genotyped at one to four of the loci Efo01 (GenBank accession number AF485776), Efo02 (AF485777), Efo03 (AF485778), and Efo04 (AF485779) based on differences between maternal and paternal genotypes. All sons in each sibship were genotyped. Sons were classified as diploid if they were heterozygous at one or more microsatellite loci. At least one daughter per sibship (when available) was also genotyped in order to confirm that heterozygotes could be detected via microsatellite polymorphisms and that microsatellite alleles were inherited biparentally. In one inbred sibship, the mother escaped midway through the season and was unavailable for genotyping; her genotype was deduced from the genotypes of her mate, one son, and one daughter.

Sibling matings in the parental $\left(\mathrm{F}_{0}\right)$ generation were classified as matched if they produced diploid sons, and unmatched otherwise. We then compared copulation durations, number of nests produced, number of offspring provisioned, and number of offspring surviving to maturity between inbred and outbred sibships, and between matched (diploid-male-producing) and unmatched (nondiploid-male-producing) sibling matings to determine whether either nestmate recognition or sexallele matching affected reproductive behavior or success.

\section{Statistical tests}

All statistical tests were performed following methods described by Zar (1999) unless otherwise noted. All $2 \times 2$ contingency tables were analyzed using a two-tailed Fisher's exact test. All $\chi^{2}$ tests with one degree of freedom were performed using the Yates correction. Before data from multiple sibships were pooled for contingency testing, a heterogeneity $\chi^{2}$ was performed to determine whether pooling this data was statistically valid.

\section{Results}

Sib-mating effects on reproductive behavior and success In all, 14 of 16 sib-mated mothers and 13 of 19 outbred mothers established nests, showing no significant difference between sib-mated and outbred mothers in tendency to nest (Fisher's exact test, $P=0.24$ ). When the 14 nesting sib-mated females were compared to the 13 nesting outbred females, there were no significant differences between sib-mated and outbred groups in copulation time, number of nests completed, number of 
nest cells provisioned, or mortality. Mean copulation time was $68.1 \pm 20.6 \mathrm{~s}$ for sibling matings and $82.2 \pm 39.3 \mathrm{~s}$ for outbred matings ( $t$-test, $P=0.27$ ). Sib-mated mothers produced on average $10.4 \pm 4.9$ nests per mother compared to $10.5 \pm 6.5$ nests per outbred mother ( $t$-test, $P=0.96)$. Sib-mated mothers provisioned an average of $38.1 \pm 17.2$ cells per mother, compared with $38.3 \pm 20.3$ cells per outbred mother ( $t$-test, $P=0.98$ ). Overall, sibmated mothers produced 426 living and 108 dead offspring, while outbred mothers produced 413 living and 85 dead offspring (Fisher's exact test, $P=0.20$ ). These results indicate that neither reproductive behavior nor overall reproductive success is affected by sibling mating as compared to outbreeding.

The relative proportions of sons and daughters were significantly different between the inbred (212 sons, 214 daughters) and outbred (129 sons, 284 daughters) groups (Fisher's exact test, $P<0.0001$ ), because of an excess of males in the inbred group when compared to the outbred control.

\section{Sex ratio comparisons}

We compared sex ratios of the two groups to determine whether these data were consistent with sl-CSD, twolocus ml-CSD, or neither. Heterogeneity $\chi^{2}$ tests on the control group $(P=0.99)$ and on the experimental group $(P=0.97)$ showed that sex ratio data, when compared among sibships within each group, were sufficiently homogeneous to be pooled for subsequent analyses.

To determine expected sex ratios under sl-CSD, we assumed that sib-mated mothers deposited the same proportion of fertilized eggs as did outbred mothers $(284 / 413=0.69)$, but that in the inbred group $25 \%$ of these fertilized eggs would develop as diploid males instead of as females. Similarly, to determine expected sex ratios under two-locus ml-CSD, we assumed that in the inbred group $6.25 \%$ of fertilized eggs would develop as diploid males instead of as females. Observed numbers of males and females (Table 1) were consistent with a null hypothesis of sl-CSD $(P=0.61)$ but not with that of two-locus ml-CSD $(P<0.0001)$. As the observed proportion of males is too large to be accounted for by two-locus CSD, and because ml-CSD models involving more than two loci predict even fewer males, the overall sex ratio difference between inbred and outbred broods supports sl-CSD but does not support the presence of any form of CSD involving two or more loci.

\section{Microsatellite DNA analyses}

Microsatellite fragment analyses showed that none of the sons in outbred sibships were diploid. In all 12 outbred sibships that produced sons, parental genotypes were sufficiently distinct so that all biparental diploids would have been detected during genotyping. Of these 12 sibships, 11 also produced daughters; genotyping of one

Table 1 Goodness-of-fit tests for sl-CSD and two-locus CSD models (expected values based on outbred sibship sex ratio)

\begin{tabular}{lccc}
\hline Category & Males & Females & $\mathrm{P}\left(\chi^{2}\right)$ \\
\hline Observed results & 212 & 214 & - \\
Expectations based on sl-CSD & 206 & 220 & 0.61 \\
Expectations based on two-locus ml-CSD & 151 & 275 & $<0.0001$ \\
\hline
\end{tabular}

daughter from each of these sibships identified each of these 11 females as a biparental diploid, confirming that microsatellite fragment analysis could identify biparental heterozygotes unambiguously. No out-of-order males were found within nests in outbred sibships.

Because of the genetic similarity between siblings, offspring of a brother-sister mating will be, on average, homozygous at more loci than will the offspring of an outcrossing. If homozygous at all marker loci, diploids cannot be distinguished from haploids. Based on the presence of at least one diploid son, six of the 14 inbred sibships were inferred to have come from parents matched at the sex alleles. In four out of these six matched inbred sibships, it was possible for a diploid offspring to be homozygous at each of the loci scored; if the mother is heterozygous at a locus and the father shares one of her alleles, then, on average, only one half of the offspring will be heterozygous at that locus and there will be a $50 \%$ chance of missing a diploid male by scoring that locus alone. If the mother is heterozygous at two loci and the father shares one allele at each, there will be a $25 \%$ chance of missing a diploid male, and, for three loci fitting this description, there will be a $12.5 \%$ chance of missing a diploid male. The chances of failing to identify true diploids within these sibships ranged from 0 (in the two sibships where parents shared no alleles at at least one locus) to 0.25 (in one sibship where the parents shared an allele at each locus and the mother was heterozygous at only two loci).

Among the 14 inbred sibships, genotyping of one or more daughters per sibship also clearly identified them as biparental diploids whenever parental genotypes were sufficiently distinct to do so. Among the six sibships produced by matched matings, 25 males developed out of normal nest order, and a total of 51 males were confirmed diploid by genotyping. The other eight inbred sibships produced no out-of-order or diploid males, and were therefore inferred to be unmatched. This is consistent with the prediction, based on sl-CSD, that $50 \%$ of sibling matings are matched at a single sex allele $\left(\chi^{2}, P=0.78\right)$. However, this $6: 8$ ratio of matched to unmatched sibling matings is not sufficient to rule out the alternative of two-locus CSD in which $25 \%$ of sibling matings are matched at both sex alleles and could therefore produce diploid males $\left(\chi^{2}, P=0.21\right)$.

Of the 25 males from matched sib-mated sibships that developed out of normal nest order, 20 were among the 51 males confirmed diploid, four could not be confirmed either haploid or diploid because maternal and paternal genotypes were sufficiently similar to produce a homozygote at all loci tested, and one was an apparent true haploid. In addition, two males from inbred sibships, in nests that contained no sisters, were out of order with respect to other males that were confirmed diploid. Neither of these males could be confirmed either haploid or diploid based on parental genotypes.

The six males that occurred out of normal nest order, but could not be confirmed either haploid or diploid, were classified as suspected diploid males. Confirmed and suspected occurrences of diploid males among the six matched inbred sibships are summarized in Table 2.

When matched sibling matings were compared to unmatched sibling matings, there were no significant differences between these subgroups in copulation duration, nests completed, cells provisioned, or mortality. 
Table 2 Diploid offspring of matched sibling matings

\begin{tabular}{lrccc}
\hline Sibship & Daughters & $\begin{array}{c}\text { Confirmed } \\
\text { diploid } \\
\text { sons }\end{array}$ & $\begin{array}{c}\text { Confirmed } \\
\text { plus suspected } \\
\text { diploid sons }\end{array}$ & $\begin{array}{c}\text { Proportion of } \\
\text { diploid sons expected } \\
\text { to be missed }\end{array}$ \\
\hline 1 & 12 & 10 & 13 & 0.125 \\
2 & 15 & 16 & 16 & 0 \\
3 & 16 & 13 & 13 & 0 \\
4 & 1 & 2 & 2 & 0.125 \\
5 & 16 & 6 & 8 & 0.125 \\
6 & 7 & 4 & 5 & 0.25 \\
\hline
\end{tabular}

Mean copulation time was $72.8 \pm 33.9 \mathrm{~s}$ for matched sibling matings and $65.1 \pm 6.7 \mathrm{~s}$ for unmatched matings ( $t$-test, $P=0.64)$. Matched mothers produced on average $12.0 \pm 4.6$ nests per mother compared to $9.2 \pm 5.0$ nests per unmatched mother ( $t$-test, $P=0.32)$. Matched mothers provisioned on average $45.2 \pm 17.2$ cells per mother, compared with $32.9 \pm 6.2$ cells per unmatched mother ( $t$-test, $P=0.20$ ). Overall, matched sib-mated mothers produced 214 living and 57 dead offspring, while unmatched sib-mated mothers produced 212 living and 51 dead offspring (Fisher's exact test, $P=0.67)$.

Proportions of sons and daughters were significantly different between the matched sib-mated group (145 sons:67 daughters) and the unmatched sibmated group (69 sons:145 daughters; Fisher's exact test, $P<0.0001$ ) due to a higher proportion of sons in the matched group. The proportions of sons and daughters (69 sons:145 daughters) in the unmatched sibling-mated group were identical to the proportions of sons and daughters (129 sons:284 daughters) in the outbred group (Fisher's exact test; $P=0.86$ )

To further rule out noncomplementary sex determination systems, it is necessary to show that diploid males are produced from fertilized eggs. Of the 51 diploid males, 32 were confirmed biparental by the presence of at least one paternal allele that was not present in the mother. The other 19 heterozygous (and therefore diploid) males could not be confirmed biparental in this manner because the mother and father shared an allele at each locus that was heterozygous in their son. However, under the assumption that sex determination is the same in the sib-mated and outcrossed groups, other possible explanations of how these diploid males arose can be ruled out. Therefore, we conclude that fertilization produced all of the observed diploid males.

\section{Weights of diploid males}

The mean adult live weight of confirmed diploid males was $52.0 \pm 16.9 \mathrm{mg} \quad(N=51)$. This was intermediate between the mean weight of females $(\bar{x}=70.1 \pm 12.2 \mathrm{mg}$, $N=478)$ and that of haploid males $(\bar{x}=38.1 \pm 10.3 \mathrm{mg}$, $N=275$ ). Weights of diploid males ranged from 16.8 to $73.4 \mathrm{mg}$, while weights of haploid males ranged from 2.4 to $66.3 \mathrm{mg}$. Weights of the six suspected diploid males ranged from 26.9 to $46.3 \mathrm{mg}$. Because this range is included within the large overlap between the known weight ranges of haploid and diploid males, we could not reliably determine the ploidy of suspected diploid males on the basis of weight.

\section{Proportion of diploid males in matched inbred broods} In order to determine whether matched inbred broods exhibited the $50 \%$ diploid male to $50 \%$ female sex ratio expected under sl-CSD, goodness-of-fit comparisons for both the sl-CSD and two-locus ml-CSD models were performed twice. For the first set of comparisons, we counted as diploid only those males confirmed diploid by heterozygosity at at least one microsatellite locus. For the second set of comparisons, we counted as diploid both genetically confirmed diploid males and the six additional males whose genotypes were inconclusive but whose out-of-order nest positions strongly suggested that they were diploid. Heterogeneity $\chi^{2}$ tests showed that sex ratio data were homogeneous across sibships from matched matings, whether considering only genetically confirmed diploid males $(P=0.55)$ or both confirmed and suspected diploid males $(P=0.72)$. Data from all six sibships were therefore pooled. The results (Table 3) were consistent with the $50 \%$ male:50\% female ratio expected under sl-CSD for confirmed diploid males $(P=0.16)$ and confirmed plus suspected diploid males $(P=0.26)$, but not consistent with the $25 \%$ diploid male to $75 \%$ female ratio expected under two-locus ml-CSD for confirmed diploid males $(P<0.0001)$ or for confirmed plus suspected diploid males $(P<0.0001)$. Therefore, slCSD is supported, while the two-locus CSD hypothesis is rejected. Since two-locus CSD was rejected because of an excess of diploid males, ml-CSD is also rejected for numbers of loci greater than two.

\section{Discussion}

These results support the hypothesis that sl-CSD is the sex determination system of E. foraminatus, and rule out the alternative hypotheses of ml-CSD and noncomplementary systems. This is in contrast to the expectation

Table 3 Goodness-of-fit tests for sl-CSD and two-locus CSD models based on matched and unmatched inbred sibship sex ratios

\begin{tabular}{|c|c|c|c|}
\hline Category & & Confirmed only & Confirmed plus suspected \\
\hline \multirow[t]{2}{*}{ Experimental results (matched matings) } & Diploid males & 51 & 57 \\
\hline & Females & 67 & 67 \\
\hline \multirow[t]{3}{*}{ Expectations based on sl-CSD } & Diploid males & 59 & 62 \\
\hline & Females & 59 & 62 \\
\hline & $P\left(\chi^{2}\right)$ & 0.16 & 0.26 \\
\hline \multirow[t]{3}{*}{ Expectations based on two-locus ml-CSD } & Diploid males & 29.5 & 31 \\
\hline & Females & 88.5 & 93 \\
\hline & $P\left(\chi^{2}\right)$ & $<0.0001$ & $<0.0001$ \\
\hline
\end{tabular}


that sl-CSD should not be present in an inbreeding species. The presence of biparental diploid males and the difference in sex ratio between inbred and outbred families together falsify alternative hypotheses of noncomplementary sex determination systems, such as genomic imprinting or genic balance. The proportions of diploid males produced by sibling-mated mothers support the presence of sl-CSD rather than ml-CSD. Although diploid males have been observed in other vespid wasps (Strassmann et al, 1994; Chapman and Stewart, 1996; Tsuchida et al, 2002), this is the first experimental study of a vespid to confirm sl-CSD by quantifying both a sex ratio shift and the production of diploid males.

These results also indicate that sib-mated females suffer no decrease in nesting tendency, fecundity, or offspring viability as compared to outbred females. Also, when considering only sibling matings, nesting tendency, fecundity, and offspring viability do not differ between matched and unmatched matings. Therefore, neither sibling mating itself nor the presence of matched sex alleles in a sibling pair affects reproductive behavior or overall fecundity. Copulation durations, while highly variable, do not differ significantly between sibling and nonsibling matings, nor between matched and unmatched sibling matings, indicating that neither nestmate recognition nor matched sex-allele recognition affects mating behavior.

Overall numbers of diploid offspring do not differ between sib-mated and outbred mothers, nor between the offspring of matched and unmatched sibling matings. This is consistent with a model in which diploid males arise from fertilized eggs that were 'intended' to produce daughters but developed as males due to sexallele matching. Our data also indicate that, in E. foraminatus, diploid male viability is comparable to that of other individuals, unlike other Hymenoptera that suffer reduced diploid male viability (Bostian, 1935; Whiting, 1961; Petters and Mettus, 1980).

Our confirmation of sl-CSD in E. foraminatus presents an apparent paradox, because the combination of inbreeding with sl-CSD will increase the number of presumably nonfunctional diploid males. Beukeboom et al (2000) proposed that loss of sl-CSD may result from selection imposed by inbreeding, because inbreeding has potentially fewer deleterious effects under noncomplementary sex determination systems. For example, chalcidoid wasps in the genera Melittobia (Schmieder and Whiting, 1947; Werren, 1993) and Nasonia (Werren, 1980, 1983; Dobson and Tanouye, 1998) are known to lack any form of CSD; these species experience inbreeding due to local mate competition (Hamilton, 1967), in which the offspring of only one or a few mothers mate among themselves in a patchy environment. Within the clade consisting of the Ichneumonoidea and the aculeate hymenopteran superfamilies, several species have also been shown to have noncomplementary sex determination. These species include two ichneumonoids in the family Braconidae, A. tabida and A. manducator, which may experience inbreeding (Beukeboom et al, 2000). An example of a non-CSD species is also known within the aculeate clade. The bethylid G. nephantidis lacks CSD; this species is also a gregarious parasitoid which, like Melittobia and Nasonia, is known to inbreed (Cook, 1993a; Luft, 1996). These examples show that non-CSD species may be distributed among taxonomic groups in which sl-CSD species also occur. If sl-CSD is indeed an ancestral condition in the Hymenoptera, these examples indicate that sex determination is evolutionarly labile and that sl-CSD can be secondarily lost.

Conversely, the presence of sl-CSD should select against inbreeding. Some braconids have sl-CSD (Speicher and Speicher, 1940; Whiting, 1943), and one such species, Bracon hebetor, has known behavioral mechanisms for sib-mating avoidance. $B$. hebetor is a gregarious parasitoid with ample sib-mating opportunities, but virgin females disperse from the natal area before becoming sexually receptive (Antolin and Strand, 1992; Ode et al, 1995). Similarly, honey bees (Apis mellifera) have sl-CSD, and siblings develop in close proximity, but mating occurs far from the natal nest and inbreeding is avoided (Koeniger, 1986).

Other behaviors and life history traits are believed to reduce the incidence and/or deleterious effects of sibmating in other sl-CSD species. For example, ichneumonids in the genus Diadegma have sl-CSD but are solitary parasitoids (Butcher et al, 2000a). Because individuals in a solitary parasitoid species are scattered throughout their environment instead of developing on the same host as do their siblings, they presumably have a lower chance of mating with siblings than do gregarious parasitoids.

Despite the apparent incompatibility between sl-CSD and inbreeding, both sl-CSD and sibling mating persist in E. foraminatus. This apparent paradox might be resolved in several ways. First, sibling mating may have benefits that outweigh its disadvantages even when sl-CSD is present. For example, by mating with her brother, a female increases her own genetic representation in her offspring (and therefore lowers the cost of sex), because many of her own maternally derived genes are also present in her brother's gametes (Cowan, 1979).

Second, observations of sibling mating among freeflying wasps at trap nests may not reflect actual levels of inbreeding. Even though Cowan (1979) estimated from field observations that approximately $40 \%$ of matings in this species take place between siblings at their natal nests, the population-wide genetic consequences of this behavior have yet to be quantified. Possibly, by mating with her brother immediately after emerging as an adult, a female gains by producing fertilized eggs as soon as possible while reducing risks associated with prolonged mate-seeking. A female could later re-mate with an unrelated male, following dispersal from the natal site. Multiple mating may reduce overall diploid male production by an already sib-mated female by making sperm from a nonrelative available (Tarpy and Page, 2002). Even though E. foraminatus females become unreceptive immediately after a single mating (Cowan, $1979,1986)$, they could become receptive again after an extended period.

Matings at nest entrances might not always be among siblings, if nonsiblings develop in the same nest cavity. Females of some wasp species are known to usurp partially completed nests of other females, resulting in mixed broods containing unrelated individuals (Krombein, 1967; Cowan, 1981). Thus, individuals mating at their natal nest entrances might in some cases be unrelated, resulting in cryptic outbreeding. 
Third, the load imposed by diploid males would be mitigated if these males have some fertility. Although diploid male hymenopterans are generally incapable of normal reproduction, El Agoze et al (1994) found that, on rare occasions, diploid males of Diadromus pulchellus father diploid daughters which are themselves capable of reproduction.

Inbreeding levels in a natural population can be quantified by measuring departures from Hardy-Weinberg equilibrium (Robertson and Hill, 1984). Genetic markers such as DNA microsatellites can potentially resolve these questions in highly mobile insects such as E. foraminatus.

\section{Acknowledgements}

Portions of this work were supported by a Western Michigan University Graduate College Dissertation Fellowship. We also thank Karim Essani, D. DeWayne Shoemaker, and Todd Barkman for their assistance with molecular techniques; Michael Stoline for his advice on statistical analyses; Jeanne Chattaway, Carrie Militzer, Wendy Tan, Talline Robadey, Jamie Lim, and Pam Laureto for other technical assistance; and Margaret Cowan, Peter Cowan, and Chris Jackson for assisting with insect rearing.

\section{References}

Adams J, Rothman ED, Kerr WE, Paulino ZL (1977). Estimation of the number of sex alleles and queen matings from diploid male frequencies in a population of Apis mellifera. Genetics 86: 538-596.

Antolin MF, Strand MR (1992). Mating system of Bracon hebetor (Hymenoptera: Braconidae). Ecol Entomol 17: 1-7.

Beukeboom LW (1995). Sex determination in Hymenoptera: a need for genetic and molecular studies. BioEssays 17: 813-817.

Beukeboom LW, Ellers J, van Alphen JJ (2000). Absence of singlelocus complementary sex determination in the braconid wasps Asobara tabida and Alysia manducator. Heredity 84: 29-36.

Bostian CH (1935). Biparental males and hatchability of eggs in Habrobracon. Genetics 20: 280-285.

Bridges CB (1925). Sex in relation to genes and chromosomes. Am Nat 59: 127-137.

Butcher RDJ, Whitfield WGF, Hubbard SF (2000a). Complementary sex determination in the genus Diadegma (Hymenoptera: Ichneumonidae). J Evol Biol 13: 593-606.

Butcher RDJ, Whitfield WGF, Hubbard SF (2000b). Single-locus complementary sex determination in Diadegma chrysostictos (Gmelin) (Hymenoptera: Ichneumonidae). J Hered 91: 104-111.

Chapman TW, Stewart SC (1996). Extremely high levels of inbreeding in a natural population of the free-living wasp Ancistrocerus antilope (Hymenoptera: Vespidae: Eumeninae). Heredity 76: 65-69.

Chilcutt CF, Cowan DP (1993). Methods for artificial rearing of solitary eumenid wasps (Hymenoptera: Vespidae: Eumeninae). Great Lakes Entomol 26: 15-19.

Cook JM (1993a). Experimental tests of sex determination in Goniozus nephantidis (Hymenoptera: Bethylidae). Heredity 71: 130-137.

Cook JM (1993b). Sex determination in the Hymenoptera: a review of models and evidence. Heredity 71: 421-435.

Cook JM, Crozier RH (1995). Sex determination and population biology in the Hymenoptera. Trends Ecol Evol 10: 281-286.

Cowan DP (1979). Sibling matings in a hunting wasp: adaptive inbreeding? Science 205: 1403-1405.
Complementary sex determination in an inbreeding wasp

JK Stahlhut and DP Cowan

Cowan DP (1981). Parental investment in two solitary wasps, Ancistrocerus adiabatus and Euodynerus foraminatus (Eumenidae: Hymenoptera). Behav Ecol Sociobiol 9: 95-102.

Cowan DP (1986). Sexual behavior of eumenid wasps (Hymenoptera: Eumenidae). Proc Entomol Soc Washington 88: 531-541.

Crozier RH (1971). Heterozygosity and sex determination in haplo-diploidy. Am Nat 105: 399-412.

Dobson SL, Tanouye MA (1998). Evidence for a genomic imprinting sex determination mechanism in Nasonia vitripennis (Hymenoptera; Chalcidoidea). Genetics 149 233-242.

Duchateau MJ, Hoshiba H, Velthuis HH (1994). Diploid males in the bumble bee Bombus terrestris. Entomol Exp Appl 71: 263-269.

el Agoze M, Drezen JM, Renault S, Periquet G (1994). Analysis of the reproductive potential of diploid males of the wasp Diadromus pulchellus (Hymenoptera: Ichneumonidae). Bull Entomol Res 84: 213-218.

Hamilton WD (1967). Extraordinary sex ratios. Science 156: 477-488

Hogge M, King PE (1975). The ultrastructure of spermatogenesis in Nasonia vitripennis (Walker) (Hymenoptera: Pteromalidae). J Submicrosc Cytol 7: 81-96.

Inaba F (1939). Diploid males and triploid females of the parasitic wasp, Habrobracon pectiophorae Watanabe. Cytologia (Tokyo) 9: 517-523.

Kerr WE, Nielsen RA (1967). Sex determination in bees (Apinae). J Apicult Res 6: 3-9.

Koeniger G (1986). Reproduction and mating behavior. In: Rinderer TE (ed) Bee Genetics and Breeding, Academic Press, Inc.: Orlando, FL pp 255-280.

Krieger MJB, Ross KG, Chang CWY, Keller L (1999). Frequency and origin of triploidy in the fire ant Solenopsis invicta. Heredity 82: 142-150.

Krombein KV (1967). Trap-nesting Wasps and Bees: Life Histories, Nests, and Associates, Smithsonian Press: Washington, DC.

Luft PA (1996). Fecundity, longevity, and sex ratio of Goniozus nigrifemur (Hymenoptera: Bethylidae). Biol Control 7: 17-23.

MacDougall C, Harbison D, Bownes M (1995). The developmental consequences of alternate splicing in sex determination and differentiation in Drosophila. Dev Biol 172: 353-376.

Mackensen O (1950). Viability and sex determination in the honey bee (Apis mellifera L.). Genetics 36: 501-509.

Ode PJ, Antolin MF, Strand MR (1995). Brood-mate avoidance in the parasitic wasp Bracon hebetor Say. Anim Behav 49: 1239-1248.

Petters RM, Mettus RV (1980). Decreased diploid male viability in the parasitic wasp, Bracon hebetor. J Hered 71: 353-356.

Robertson A, Hill WG (1984). Deviations from Hardy-Weinberg proportions: sampling variances and use in estimation of inbreeding coefficients. Genetics 107: 703-718.

Ross KG, Fletcher JC (1985). Genetic origin of male diploidy in the fire ant, Solenopsis invicta (Hymenoptera: Formicidae), and its evolutionary significance. Evolution 39: 888-903.

Schmieder RG, Whiting PW (1947). Reproductive economy in the chalcidoid wasp Melittobia. Genetics 32: 29-37.

Smith SG, Wallace DR (1971). Allelic sex determination in a lower hymenopteran, Neodiprion nigroscutum Midd. Can J Genet Cytol 13: 617-621.

Speicher BR, Speicher KG (1940). The occurrence of diploid males in Habrobracon brevicornis. Am Nat 74: 379-382.

Stahlhut JK, Cowan DP, Essani K, Shoemaker DD, Barkman TJ (2002). Microsatellite markers for the wasp Euodynerus foraminatus (Vespidae: Eumeninae). Mol Ecol Notes 2: 467-468.

Strassmann JE, Hughes CR, Turillazzi S, Solis CR, Queller DC (1994). Genetic relatedness and incipient eusociality in stenogastrine wasps. Anim Behav 48: 813-821.

Tarpy DR, Page RE (2002). Sex determination and the evolution of polyandry in honey bees (Apis mellifera). Behav Ecol Sociobiol 52: 143-150. 
Tsuchida K, Nagata N, Kojima J (2002). Diploid males and sex determination in a paper wasp, Polistes chinensis antennalis (Hymenoptera, Vespidae). Insect Soc 49: 120-124.

Werren JH (1980). Sex ratio adaptations to local mate competition in a parasitic wasp. Science 208: 1157-1159.

Werren JH (1983). Sex ratio evolution under local mate competition in a parasitic wasp. Evolution 37: 116-124.

Werren JH (1993). The evolution of inbreeding in haplodiploid organisms. In: Thornhill NW (ed) The Natural History of Inbreeding and Outbreeding, University of Chicago Press: Chicago pp 42-59.

Whiting AR (1961). Genetics of Habrobracon. Adv Genet 10: 295-348.
Whiting PW (1943). Multiple alleles in complementary sex determination of Habrobracon. Genetics 28: 365-382.

Whiting PW (1960). Polyploidy in Mormoniella. Genetics 45: 949-970.

Woyke J, Skowronek W (1974). Spermatogenesis in diploid drones of the honeybee. J Apicult Res 13: 183-190.

Yamauchi K, Yoshida T, Ogawa T, Itoh S, Ogawa Y, Jimbo S et al (2001). Spermatogenesis of diploid males in the formicine ant, Lasius sakagamii. Insect Soc 48: 28-32.

Yokoyama S, Nei M (1979). Population dynamics of sexdetermining alleles in honey bees and self-incompatibility alleles in plants. Genetics 91: 609-626.

Zar JH (1999). Biostatistical Analysis, 4th edn. Prentice-Hall, Inc.: Englewood Cliffs, NJ. 